\author{
SAGGI - ESSAYS \\ CUORI SPEZZATI. \\ L'ODIO E LA VIOLENZA NARRATI A'TTRAVERSO \\ LA LETTERATURA PER L'INFANZIA E PER RAGAZZI \\ di Maria Teresa Trisciuzzi
}

La ricerca del sé e della propria identità viene vissuta dai bambini e dai ragazzi come una grande fatica, avvertendola sia dentro che fuori la famiglia e la scuola. I conflitti verbali e fisici, attraverso atti di bullismo e di cyberbullismo (Chadwick, 2014), segnano i giovani, sia che essi siano vittime, sia che siano bulli (Viljoen, O’Neill \& Sidhu, 2005; Burgio, 2012; Trisciuzzi, 2018b). L'odio e la violenza appresi tra le pareti domestiche, tra le mura scolastiche e nei viaggi sui social, si introducono in maniera feroce e crudele nella vita dei giovani, cambiandone il modo di vivere e di comunicare con gli altri. La diffusione di odio e di violenza fisica e psicologica può essere definita "inquinamento emotivo", attraverso cui i sentimenti e le emozioni dei bambini, dei ragazzi e di chi li circonda vengono contaminati (Contini, 1992; Dato, 2004; Loiodice, 2004; Fabbri, 2008). Lo sguardo e i cuori delle infanzie narrate nella letteratura per l'infanzia restano aggrappati alla speranza e alla bellezza, trasmettendole ai lettori, avidi oggi di trovare orizzonti e sogni da realizzare.

Il presente contributo esamina le rappresentazioni letterarie e filmiche in cui disagio e violenza sono protagonisti, la loro interpretazione pedagogica e la rilevanza nella letteratura per l'infanzia.

The search for themselves and their own identity by children and young people is experienced as a great effort, feeling it both inside and outside the family and the school. Verbal and physical conflicts, through acts of bullying and cyberbullying (Chadwick, 2014), mark young people, whether they are victims or bullies (Viljoen, O’Neill, Sidhu, 2005; Burgio, 2012; Trisciuzzi, 2018b). Hate and violence learned in the home, within the school walls and 
on 'social trips', arrive ferociously and cruelly in young people's lives, changing the way they live and communicate with others.

The spread of hatred and physical and psychological violence can be defined as "Emotional Contamination", through which the feelings and emotions of children, young people and those around them are affected (Contini, 1992; Dato, 2004; Loiodice, 2004; Fabbri, 2008). The eyes and hearts of the children narrated in the children's literature remain clinging to hope and beauty, transmitting them to readers, eager today to find horizons and dreams.

This paper examines the literary and filmic representations in which suffering and violence are the protagonists, their pedagogical interpretation and their relevance in children's literature.

\section{Una premessa}

Metafore d'identità, i protagonisti e le protagoniste della letteratura per l'infanzia sono da sempre frontalieri in bilico sul limen tra il reale e l'immaginario conosciuto dai giovani lettori. Questi varcano oggi soglie attraverso le quali l'odio e la violenza fisica e psicologica si propagano come una macchia d'olio nera su una superficie inizialmente pura e incontaminata dai pregiudizi e gli stereotipi, espandendosi sempre più.

Il pensiero ecologico (Bateson, 2000) sostiene infatti la nascita di una nuova narrazione, quella dell'empatia: una dimensione che fa leva sui sentimenti di immedesimazione nelle vite esperienziali degli altri, divenendo oggetto di un racconto socialmente e culturalmente condiviso. Partendo da questo concetto, come afferma Maurizio Fabbri (2014), «l'empatia sembra prendere il posto della speranza, quale unico dono superstite nel vaso di Pandora, dopo che la decisione di scoperchiarlo ne fece uscire tutti i mali del mondo» (p. 9). Un'empatia contaminata, verrebbe da dire un inquinamento emotivo, attraverso cui i sentimenti e le emozioni dei bambini, dei ragazzi e di chi li circonda vengono contaminati da una diffusione di aggressività ed ostilità. Una empatia «costretta a convivere con le peggiori forme di crudeltà, l'egocentrismo e l'anestesia 
emotiva, ma pur sempre un sentimento che si evolve e si radica e si potenzia, al punto da risultare necessario ai fini della sopravvivenza della specie» (Fabbri, 2014, p. 9).

La diffusione di curricula formativi colmi di rappresentazioni sociali stereotipate «ha impedito per più di due secoli di realizzare in ambito scolastico quel percorso di riconoscimento individuale che solo negli ultimi anni ha portato bambini e bambine, ragazzi e ragazze a pensarsi diversi, ma uguali per valore, e capaci di intrattenere relazioni non più contaminate da falsi modelli del maschile e del femminile» (De Serio, 2015, p. 65). È importante quindi oggi dare voce e riconoscimento ai vissuti autentici sia maschili che, soprattutto, femminili e alla loro specificità. Ascoltando la voce al femminile narrata attraverso i romanzi della letteratura per l'infanzia si può riconoscere un desiderio di autonarrazione. Le bambine e le ragazze, ormai fuori dalle mura costrittive ottocentesche, che le indirizzavano a un destino di piccole vestali domestiche, disvelano la propria disobbedienza e divergenza rispetto ai canoni pre-imposti del passato.

Questo significa mettere a disposizione delle donne, fin dai primi anni di vita, strumenti per conoscersi e valorizzarsi, interpretare e interpretarsi nelle proprie differenze, determinate innanzitutto dall'appartenenza di genere, al fine di disoccultare i meccanismi della discriminazione dei ruoli - maschili e femminili - oggi presenti come e forse più di ieri. $\mathrm{O}$ forse più pervicaci rispetto a ieri proprio perché occultati da immagini di «falsa emancipazione, che ancora una volta finiscono per rinchiudere il genere femminile in stereotipi che non corrispondono alla pluralità dell'esperienza femminile e che non consentono al genere maschile di cogliere la ricchezza di relazioni tra uomini e donne non più succubi di logiche di predominio e pronti invece a cogliere la positività di un rapporto che si fa paritario proprio attraverso la differenza» (Loiodice, Pinto Minerva, 2015, pp. XI-XII).

I giovani lettori, leggendo per emanciparsi (De Serio, 2015), guardano se stessi come attraverso un filtro, prendendo le distanze e rielaborando le proprie esperienze. Proprio per questo non hanno bisogno di storie edulcorate o iperealistiche, ovvero di storie in cui tutto viene esasperato ed esagerato, ma di storie con protagonisti autentici che hanno problemi veri, dolori effettivi, vite reali. 
Si nota come, attraverso la lente della letteratura per l'infanzia e per ragazzi, appaiono modelli di genere differenti tra femmine e maschi che riguardano il tema del bullismo.

Il cyberbullismo, ovvero il "bullismo online", può essere più diffuso del "bullismo offline", tuttavia l'attenzione al cyberbullismo va posta sullo stesso piano del bullismo fisico e offline. Varie ricerche (Viljoen, O’Neill \& Sidhu, 2005; Field, Kobler, Crothers \& Hughes, 2009; Chadwick, 2014; Chan \& Wong, 2019) affermano che la maggior parte del cyberbullismo è praticato da ragazze ed è coerente con i tipi di bullismo indiretto. Le ragazze, infatti, sono spesso coinvolte nel cyberbullismo sia come bersagli (e perciò come vittime), sia come parte attiva - si parla in tal caso di Mean Girls (McClung, 2006; Brock, Oikonomidoy, Wulfing, Pennington \& Obenchain, 2014; Walsh, Sekarasih \& Scharrer, 2014; Dentith, Redmon Wright \& Coryell, 2015). Rispetto ai maschi, tra i quali predomina l'aggressione relazionale diretta/fisica, emerge che le ragazze hanno maggiori probabilità di essere protagoniste e vittime di cyberbullismo, ovvero di quel bullismo informatico che si attua tramite messaggi di testo e telefonate. Il "bullismo femminile" (Trisciuzzi, 2018b) va oltre il qui e ora, prospettandosi come un fenomeno sempre più in crescita, oggi anche attraverso le nuove tecnologie. Nel mondo femminile il bullismo è rivolto alle compagne con maldicenze, pettegolezzi, fino ad arrivare all'esclusione dalla cerchia sociale.

Si tratta, perciò, come afferma Daniela Dato (2016), «di ri-cordare il valore della dimensione emotiva, ovvero, etimologicamente, di riportarla al cuore, di non desistere dal far prendere consapevolezza agli uomini e alle donne di oggi di quanto l'incontro tra ragione ed emozione possa rivelarsi sfida di sviluppo ed emancipazione» (p. 241).

Diventa significativo quindi coltivare una pedagogia della reciprocità - evidenziando l'importanza della dimensione intersoggettiva dello sviluppo umano - basata su quei beni relazionali quali la comprensione, la compassione, il confronto, il dialogo, l'empatia, gemme nate dal terreno fertile della condivisione e dell'ascolto.

Strategia per contrastare l'odio e la violenza che contaminano oggi la nostra società è promuovere ed esercitare maggiormente la rilevante competenza emotiva e sociale dell'empatia. Questa ci con- 
sente di prendere consapevolezza non solo di noi stessi ma anche dell'altro. Il soggetto presente in divenire, costituito da un mosaico di molteplici sfaccettature comprendenti la dimensione genetica, culturale e sociale, grazie all'approfondimento delle competenze emotive e sociali, può resistere ai rischi di disumanizzazione, dell'omologazione dello sguardo, orientandosi invece verso una riflessione e un pensiero critico e propositivo, non conformato agli stereotipi e ai pregiudizi che i "discorsi d'odio" tendono a creare. Infatti, «se l'empatia è un sentire dal di dentro, essa è pure dialogo, è capacità di prestare attenzione all'altro» (Dato, 2016, p. 243), cercando di aprire con il diverso da sé un canale comunicativo, ovvero con il suo mondo e la sua storia. Le emozioni si coniugano con l'importante tema dell'identità e dell'alterità, promuovendo una visione di rispetto e di apertura verso "l'altro". E, come direbbe Andrea Camilleri, conducendo la riflessione ancora oltre: «Ma perché l'altro è diverso da me? L'altro non è altro che me stesso allo specchio» ${ }^{1}$.

Aprendo le porte a un'educazione alle emozioni e al pensiero riflessivo, lo sguardo si dirige anche sull'importanza dell'educazione estetica intesa come educazione alla sensibilità e alla bellezza, considerata come capacità emotiva opposta al conflitto e alla discordia. Tendere verso una educazione alle emozioni significa quindi sottolineare l'unione di due dimensioni, ovvero quella del cuore e quella della ragione, da sempre considerate incompatibili. Ed è proprio l'unione di queste due sfere che può portare ad una riconciliazione del sé con l'altro.

\section{L'età dell'incertezza}

L'adolescenza è, come afferma Franco Cambi (2015), «un'età complessa e preziosa. Un'età di scoperte, di tensioni, di risveglio interiore, di trasformazione fisica e psicologica» (p. 16), un'età di conflitti: «chi è fuori dal gruppo, chi è diverso, diventa spesso il

1 Dall'intervista ad Andrea Camilleri, condotta da Fabio Fazio nel programma "Che tempo che fa", per presentare "Conversazione su Tiresia", il suo nuovo film. Consultabile su RaiPlay. 
'capro espiatorio': è oggetto di atti di bullismo, di mobbing, di derisione; non gli si dà tregua. Il negativo si condensa tutto sui capri espiatori e il gruppo e l'individuo si liberano così del suo peso» (Cambi, 2015, p. 16).

La crescita può essere considerata una condizione che si compie attraverso la perdita dell'innocenza di fronte al disvelarsi del terribile segreto che nasconde l'essere adulti: la morte. La morte ha, prima di tutto, un fortissimo valore simbolico poiché davanti ad essa svanisce qualsiasi speranza di avere un futuro, perché già formato e prestabilito oppure perché considerato mera illusione (Bosio, 1996; Faeti, 2001b; Scabello Garbin, 2014). Come afferma David Almond (2004): «la presenza della morte enfatizza il miracolo della vita, il buio esiste perché esiste la luce [...]. Ma sappiamo che poco più in là ci può essere la morte. È il rapporto tra le due cose che dà loro un senso» (p. 17).

Il tema del suicidio o del tentato suicidio viene affrontato anche nella letteratura per l'infanzia: ad esempio lo si trova in Thornhill di Pam Smy, la storia di due bambine narrata su due livelli, uno letterale e uno figurativo. Mary vive negli anni Ottanta a Thornhill, un orfanotrofio femminile che sta per essere chiuso; Ella, invece, è una ragazza che nel 2017 si trasferisce con il padre accanto all'edificio, ormai abbandonato da tempo. Il romanzo, dichiarato omaggio a Brian Selznick e alle atmosfere de Il giardino segreto della scrittrice Frances Hodgson Burnett, narra le storie delle due ragazzine. Al di là del tempo e dello spazio, sulla pagina le due storie si incontrano e si intrecciano, l'una col testo del suo diario, l'altra con le immagini in bianco e nero.

Nella vita reale, come attraverso le pagine del romanzo/graphic novel di Pam Smy, il suicidio - o il suo tentativo - è purtroppo presente tra $i$ giovani. Che il suicidio in adolescenza abbia un significato specifico, ovvero che sia correlato con le radicali problematiche della fase evolutiva che il soggetto sta attraversando, è confermato dalle ricerche scientifiche sul caso (Ladame, 1987; Pietropolli Charmet, 2004; Barone, 2009; Hinduja \& Patchin, 2010).

Il tema del suicidio ritorna anche in alcuni fumetti e romanzi della letteratura per l'infanzia e per ragazzi, come Jane, la volpe \& io, 
scritto da Isabelle Arsenault e illustrato da Fanny Britt. Il picturebook/graphic novel, intriso delle emozioni della protagonista, permette di entrare in piena sintonia con lei, cogliendo tanto la dolcezza quanto la vulnerabilità del suo carattere. Lo spessore emotivo che traspare dalle pagine della storia ricorda Thormbill, essendo entrambe le storie accomunate dal bianco e nero delle immagini. Il colore sopraggiunge contemporaneamente alla felicità e alla serenità conquistata dalla protagonista, grazie alla relazione positiva con i pari e la nuova fiducia trovata in se stessa, superando i pensieri di morte.

Si nota che l'età è un fattore determinante, e il suicidio o il suo tentativo sono correlati con alcune condizioni familiari e sociali che vengono, pertanto, considerate fattori di rischio. Gli adolescenti, narcisisticamente fragili, perseguitati dalla paura di perdere la faccia, dai confini del sé ancora molto incerti e mal segnati perché dipendenti ancora dagli altri, dal giudizio degli altri, dalle missioni affidate, dai riconoscimenti non ufficiali e appunto per ciò molto importanti, arrivano talvolta a vergognarsi da morire (Burgio, 2018).

L'odio e l'intolleranza possono colpire ferocemente i giovani, e la letteratura per l'infanzia e per ragazzi parla loro attraverso storie metaforiche, in cui si riconoscono e attraverso le quali possono rivedere se stessi, trovando nuovi percorsi possibili di speranza e di futuro possibile.

Scritto nel 1976 da Katherine Paterson, e da cui è stato tratto l'omonimo film uscito nel 2007, nel romanzo Un ponte per Terabithia viene narrata la storia dell'amicizia tra un ragazzo e una ragazza, Leslie e Jess che, grazie alla loro fervida immaginazione, costruiscono un loro "giardino segreto", un mondo incantato nel verde dietro casa. Un luogo magico in cui combattono i giganti, simili alle gigantesche avversità che devono superare ogni giorno nella realtà. Il gigante cattivo veste infatti i panni della bulla della scuola, Janice Avery, che prendeva di mira i bambini più piccoli. La ragazza, la mattina, qualche volta si piazzava davanti alla porta del bagno delle femmine e costringeva le bambine più piccole a consegnare i loro denari, pena il divieto di accesso al bagno. Fino a quando un giorno Leslie trova Janice nel bagno mentre stava piangendo e ne parla con Jess: «Adesso capisco perché Janice abbia tutti quei problemi 
nei rapporti con gli altri». [...] «Lo sapevi che suo padre la picchia? [...] Il tipo di botte che fa andare in prigione la gente, ad Arlington» (Paterson, 2007, p. 126).

I giganti, un po' come le creature selvagge di Maurice Sendak, ritornano anche all'interno del fumetto I kill Giants, scritto da Joe Kelly e disegnato da J. M. Ken Niimura, uscito negli Stati Uniti per la prima volta nel 2010 e trasposto successivamente nel 2017 nell'omonimo adattamento cinematografico, diretto da Anders Walter. Nel film, Barbara, la coraggiosa protagonista, combatte una guerra contro dei giganti, o per meglio dire, contro i suoi personali "mostri giganteschi". Barbara vive in una piccola cittadina insieme al fratello e la sorella che, appena ventenne, tenta di svolgere il complesso ruolo di capofamiglia, ma con molta difficoltà, data sia la situazione che la sua giovane età. Abbandonati dal padre, i ragazzi si ritrovano a fronteggiare psicologicamente ed economicamente la grave malattia della madre. La protagonista trascorre le giornate in compagnia della propria fantasia, costruendo trappole destinate ad arrestare l'arrivo delle spaventose e terrificanti creature portatrici di caos.

I Kill Giants si presenta agli occhi del lettore/spettatore come una storia caratterizzata da una trama fantastica attraversata dal delicato tema dei riti di passaggio (Van Gennep, 1981) dal periodo dell'infanzia a quello più maturo e consapevole dell'adolescenza. Joe Kelly, insieme ai caratteristici disegni di Ken Niimura, ci descrive le difficoltà dell'infanzia, incompresa dal mondo adulto, ad affrontare le problematiche dello sviluppo in assenza di punti di riferimento. Barbara, molto spesso abbandonata a se stessa, tenta di far fronte alla malattia della madre rifugiandosi in un mondo fittizio; infatti è solo alla fine del film che si comprende la natura dei giganti: essi non sono altro che le forti paure e le violenti angosce della quotidianità. Tuttavia, l'adolescenza porta con sé anche una diversa forma di consapevolezza che induce la protagonista ad adottare un nuovo punto di vista, attraverso cui riesce a "combattere" le proprie inquietudini e le proprie ansie. Il disagio della stravagante protagonista viene amplificato dalla difficoltà nel rapportarsi 
con i propri coetanei, in un sistema sociale in cui ogni forma di diversità è respinta.

I "mostri giganteschi" ritornano anche nel romanzo Sette minuti dopo la mezzanotte (A Monster Calls), pubblicato nel 2011, scritto da Patrick Ness, tratto da un'idea originale di Siobhan Dowd ed illustrato da Jim Kay. «Il mostro si presentò poco dopo la mezzanotte. È così che fanno» (Ness, Dowd, 2012, p. 11). Questa potente storia, che esplora la paura, il dolore, la perdita e la morte, ci mostra le esperienze scolastiche del protagonista. Come affermano i ragazzi di Mare di libri: «Ness, ispiratosi ad un soggetto della Dowd rimasto incompiuto, racconta il travaglio di un adolescente, Conor, alle prese con una prova terribile, la malattia della madre, attraverso il racconto della sua crescita umana in bilico tra fantasia e realtà, rimozione e presa di coscienza: la paura del mostro che arriva sette minuti dopo la mezzanotte e il puro terrore per l'incubo vero e proprio, il più terribile dei sogni. Un ragazzo alla ricerca della verità, forse l'unica medicina in grado di curare la sua anima» ${ }^{2}$. La carriera artistica della Dowd, purtroppo, fu tragicamente interrotta dalla sua morte prematura a causa di un tumore. Il Trust ${ }^{3}$ che ha fondato nei suoi ultimi giorni di vita continua l'opera di portare libri a chi non può avere accesso ad essi e alla lettura.

\section{Ascoltando il cuore delle narrazioni familiari}

Fra $i$ tanti elementi che potrebbero aiutare i ragazzi e le ragazze - vittime o carnefici - nell'arduo compito della crescita c'è sicuramente la famiglia, che riveste un ruolo decisamente importante (Fratini, 2015).

2 https://maredilibri.it/rubriche/il-colpo-di-fulmine/sette-minuti-dopo-lamezzanotte/ [20.10.2019].

${ }^{3}$ Come afferma Ness, Siobhan Dowd «era una donna che ha lavorato per organizzazione di difesa dei diritti umani, che ha costituito il Siobhan Dowd Trust in modo che dopo la sua morte il ricavato dei suoi libri potesse aiutare i bambini che ne hanno bisogno» (Ness, 2018, p. 106). 
Sempre più spesso, nella letteratura per ragazzi, il punto di partenza della narrazione è una famiglia assente o insoddisfacente (Trisciuzzi, 2018a), incapace di rispondere ai problemi e alle richieste degli adolescenti protagonisti delle storie: una cosiddetta famiglia disfunrionale. Succede nel romanzo della scrittrice francese MarieAude Murail (Trisciuzzi, 2016), Nodi al pettine, in cui viene narrata la storia del quattordicenne Louis che, dovendo fare uno stage, accetta senza pensare la proposta della nonna e comincia a lavorare al salone di acconciature da lei frequentato. Louis va contro il volere del padre che rifiuta la scelta del figlio, non desiderando per lui "quella roba", uno stage che, riassunto nel suo pensiero, era: «un salone di parrucchiera, in fatto di moralità si poteva trovare di meglio» (Murail, 2011, p. 53). Le parole del padre sono per Louis come "un chiodo nel cuore", infatti se Louis avesse trovato quelle giuste per spiegarsi, avrebbe voluto dirgli che «stava effettivamente lavorando per il proprio avvenire. Visto che voleva fare il parrucchiere. Si portò le mani al cuore. Avrebbe voluto strapparsi qualcosa» (Murail, 2011, p. 128). La famiglia tace e omette la verità, l'amore di Louis per quel lavoro e quella professione in cui si vede proiettato nel futuro. La violenza del padre esplode nel momento in cui scopre che suo figlio continua a frequentare il salone, mandandolo al pronto soccorso con un pugno di rabbia feroce. Stravolto e inconsapevole della violenza che albergava dentro di sé, il padre di Louis si vergogna di ciò che ha fatto e crolla: le barriere borghesi erette durante la sua vita cedono, "i nodi vengono al pettine" e per il padre si apre una porta sulla vita del figlio, una riconciliazione familiare e affettiva. I cuori spezzati dai pregiudizi, dall'odio e dalla violenza si riparano.

L'extra-familiare diventa un altrove in cui i protagonisti possono essere diversi da come sono nel loro Qui, ovvero nel loro ambiente domestico, in cui sono presenti adulti che li conoscono, ma da cui non sono compresi appieno. La scuola, ad esempio, appare simbolo di separatezza e di isolamento, un luogo dove sembra possibile definire la propria identità. Solo quando il bullismo entra a far parte della vita scolastica, definire se stessi diventa una sfida difficile per molti, soprattutto quando si deve lottare per la propria identità. 
È proprio nella famiglia o nelle istituzioni, che dovrebbero essere i luoghi più sicuri ed accoglienti, che si nota come si verifichino spesso i divari più drammatici: basta pensare agli orribili genitori di Matilde (Dahl, 1988), persone ignoranti, bugiarde e imbroglione, talmente ottuse da non rendersi nemmeno conto della grande intelligenza della figlia. Basti pensare alla madre della protagonista, la signora Dalverme, emblema della cattiva madre.

Le donne, le madri rappresentate nei romanzi di Dahl «sono il riflesso di un'assenza dei genitori e sottolineano la necessità di trovare una figura parentale che li aiuti a crescere» (Filograsso, 2012, pp. 196-197). Oppure basta ricordare la spaventosa direttrice Spezzindue, che "ama" i ragazzini della sua scuola al punto da lanciarli lontano in aria come se fossero martelli olimpionici, affermando che «domare una mocciosa perversa è come cercare di schiacciare un moscone su una cacca. Cerchi di colpirlo e quello è già volato via. Che cosa disgustosa, le bambine. Per fortuna io non sono mai stata una bambina» (Dahl, 2003, p. 81).

$\mathrm{E}$, per fortuna, c'è un'insegnante diversa, la signorina Dolcemiele, un'adulta che si sa avvicinare alla sensibilità infantile, che potremmo definire come "insegnante emotiva". L'eccezionale ed affettuoso legame di Matilde con la sua maestra costituisce il nucleo emotivo della storia e, alla fine, la protagonista decide di abbandonare la sua famiglia disfunzionale per vivere con la sua nuova amica adulta che le è molto più congeniale.

La scrittrice Melania Mazzucco, nella sua Introdurione al romanzo Ascolta il mio cuore di Bianca Pitzorno, nota e amata scrittrice contemporanea, afferma che «la cosa più importante, allora come oggi, erano i rapporti tra le persone. E nel caso dei bambini, i rapporti con i coetanei da un lato, e dall'altro col mondo spesso incomprensibile degli adulti» (2012, p. 6).

Ambientato nel periodo del secondo dopoguerra, il romanzo è legato alle rappresentazioni della ricerca del riscatto sociale, nel pesante ricordo delle separazioni e delle difficoltà affrontate dalle famiglie negli anni precedenti. Diario di un anno scolastico, come l'illustre predecessore Cuore, il romanzo è una cronaca degli eventi, talvolta buffi, altre gravi, di una classe di quarta elementare. Ascolta 
il mio cuore è colmo di narrazioni irriverenti scritte in prima persona dalla protagonista, Prisca Puntoni, i cui scritti svolgono il ruolo dei racconti mensili di De Amicis, «sovvertendone l'intento edificante» (Ivi, p. 6). Prisca è una bambina che «scrive; scrive di tutto, temi, cronache, invettive, e i suoi testi, fiabe, commenti, narrazioni che siano, fungono da controcanto liberatorio nei confronti di un combattimento che non concede tregue» (Casella, 2006, p. 206).

Le storie sono scritte dalla bambina come denuncia dei fatti a cui assiste a scuola, così da portare la sua vendetta contro le prepotenze su carta, dispotismi da parte della maestra che le fanno sobbalzare il cuore: «Prisca si agitava sul banco come un'anguilla. Ascolta il mio cuore! - bisbigliò afferrando la mano di Elisa e premendola sul petto. - Sta per scoppiare. BUM BUM BUM» (Pitzorno, 2012, p. 47).

Prisca e le sue amiche e compagne di classe Elisa e Rosalba si trovano a combattere, con tutta l'immaginazione in loro possesso e con ogni mezzo che riescono a trovare, la loro maestra Argia Sfor$\mathrm{ra}^{4}$, soprannominata dalle bambine Arpia Sferza, e i suoi soprusi. L'Arpia è un «terrificante esempio di educatrice davvero seguace di una tradizione in cui la sferza sui deboli e l'untuoso ossequio verso i potenti sono le prospettive di fondo di un atteggiamento e di un comportamento che, nel loro infame e coerente procedere, assumono quasi l'aspetto di una velenosa missione» (Faeti, 2001a, p. 46). Coraggiosa eroina bambina, Prisca non riesce a resistere davanti ai soprusi e lotta insieme a Elisa e Rosalba contro le ingiustizie con forza, insegnando a chi segue la sua storia una cosa fondamentale, ovvero che non ci si deve arrendere di fronte alle violenze e alle prevaricazioni, sia che provengano dai pari, che dagli adulti.

\section{Conclusioni}

Il compito del "saper essere", rientrando anche tra le competenze della scuola, può essere considerato come possibilità di offri-

${ }^{4}$ L'Argia è un tipo di ragno sardo velenoso il cui morso, in alcuni casi, può portare alla morte. 
re all'infanzia una occasione, aprendo le porte alle scelte e alle possibilità sul presente e sul futuro, e creando una "interdipendenza dialettica" (Frabboni, 2012; Baldacci \& Pinto Minerva, 2015) tra logos ed emozione, consentendo ai bambini perciò di appropriarsi di modi di pensare altri, indirizzandoli verso una comprensione più complessa di sé e del mondo. Il rapporto educativo, infatti, è sempre un rapporto che chiama in causa gli affetti: non c'è rapporto in cui non sia prevista una componente emotiva.

Mariagrazia Contini $(1992 ; 2002)$ sostiene quanto ormai sia indispensabile, all'interno di programmi di formazione, una educazione all'ascolto e al riconoscimento delle emozioni, in una prospettiva formativa e di riflessione, attraverso il dialogo e il confronto consapevole, anche grazie alla costruzione della presenza di setting simbolici e non, ovvero spazi riservati all'apprendimento della comunicazione emotiva. Emerge quindi la significante figura dell'“insegnante affettivo" (Cambi, 1994; Goleman, 1996; Rossi, 2002), o anche "insegnante emotivo" (Dato, 2004; Fabbri, 2008; Corsi, Fabbri \& Riva 2016; Dato, 2019), che veste gli abiti di facilitatore dei processi di apprendimento, valorizzando i propri allievi, ascoltandone i bisogni emotivi nelle loro molteplici forme.

Come già notava Giovanni Maria Bertin (1975), la necessità di promuovere nell'insegnante la capacità di aiutare lo studente a riconoscere i propri squilibri emotivi, orientando la sua formazione da un punto di vista sia intellettuale che sociale e affettivo, è ormai indispensabile. Dunque: «linsegnamento deve ridiventare non più solamente una funzione, una specializzazione, una professione, ma un compito di salute pubblica» (Morin, 2000, p. 106).

In un'età in cui il desiderio di essere accettati è peculiare, in una società, come quella attuale, in cui le bambine sono sempre più precoci (Contini \& Demozzi, 2016), urge un cambiamento nelle pratiche di vita: un ritorno all'essenziale, al profondo, alla riflessione, alla dimensione dell'autentico. Ed è fondamentale per questa trasformazione antropologica riuscire a riappropriarsi del proprio tempo, sostituendo l'idea del tempo come "guadagno" con quella di tempo come "occasione" per ritornare a sé, con sé, in armonia. Scalzare dunque il mito della velocità per accogliere quello della 
lentezza (Augè, 2016), intesa come possibilità di approfondimento interiore, delle proprie emozioni, ed esteriore dei propri sensi per godere di tutto quello che ci circonda, per riuscire a dare importanza alle cose vere, e saper godere della gioia data dalle piccole cose. Si tratta di una sfida non facile, ma possibile a patto di aprirsi all'ascolto e al rispetto profondo dell'altro, specialmente dei bambini, che con le loro domande riescono ad evidenziare le contraddizioni e l'iniquità della logica imperante degli adulti di oggi.

Impiegare tempo a parlare non significa "perdere tempo", ma rappresenta la premessa indispensabile per una corretta relazione educativa: non si può prescindere, infatti, dalla reciproca conoscenza per creare un clima sociale positivo ed è possibile costruire tale reciprocità solo ascoltando e conversando con l'infanzia.

L'ascolto è una delle esperienze più significative e fondamentali, rappresentando la premessa di quell'empatia necessaria per fare dell'insegnamento una relazione d'aiuto nella crescita e nello sviluppo educativo. Solo trasformando il desiderio in progetto, in un percorso di orientamento verso il futuro, il docente diventa un insegnante emotivo, vestendo questo babitus e una nuova forma mentis professionale che apre a un atteggiamento attivo e propositivo, diretto a una pedagogia relazionale, «attenta all'altro e all'oltre» (Dato, 2019, p. 128), sensibile all'ascolto e alla riflessione problematicista.

\section{Bibliografia}

Acone L. (2018). Bambini e ragazzi tra bande e paranze. Pedagogia della narrazione a Sud dell'infanzia. Lecce: Pensa MultiMedia.

Almond D. (2004). Intervista a David Almond. Fuorilegge, 1, 17-18.

Augè M. (2016). Prendere tempo. Un'utopia dell'educazione. Conversazione con

Filippo La Porta. Roma: Castelvecchio Editore.

Baldacci M. \& Pinto Minerva F. (a cura di) (2015). Razionalità, educazione, real-

tà sociale. Studi sulla pedagogia di Franco Frabboni. Milano: FrancoAngeli.

Barone P. (2009). Pedagogia dell'adolescenza. Milano: Guerini.

Bateson G. (2000). Verso un'ecologia della mente. Milano: Adelphi.

Bauman Z. (2014). La società dell'incertezza. Bologna: Il Mulino.

Bertin G. M. (1975). Educazione e alienazione. Firenze: La Nuova Italia. 
Bosio M. (1996). Ogni ragazzo è un’isola. In E. Beseghi (a cura di). L'isola misteriosa. Adolescenz̧a, quaderni di letteratura per l'infanz̧ia. Milano: Mondadori.

Brock C.H., Oikonomidoy E.M., Wulfing K., Pennington J.L. \& Obenchain K.M. (2014). "Mean Girls" Go to College: Exploring Female-Female Relational Bullying in an Undergraduate Literacy Methods Course. Peace and Conflict: Journal of Peace Psychology, 20(4), 516-535.

Burgio G. (2012). Adolescenza e violenza. Il bullismo omofobico come formazione alla maschilità. Milano-Udine: Mimesis.

Burgio G. (2018). Comprendere il bullismo femminile. Genere, dinamiche relazionali, rappresentazioni. Milano: FrancoAngeli.

Cambi F. (1994). I silenzi della teoria: il nascosto e l'impensato. In F. Cambi \& S. Ulivieri (a cura di). I silenæi nell'educarione. Studi storicopedagogici in onore di Tina Tomasi. Firenze: La Nuova Italia.

Cambi F. (2015). Omofobia a scuola. Una classe fa ricerca. Pisa: ETS.

Casella M. (2006). Le voci segrete. Itinerari di iniziazione al femminile nell'opera di Bianca Pitzorno. Milano: Mondadori.

Chadwick S. (2014). Impact of Cyberbullying, Building Social and Emotional Resilience in Schools. Sydney: Springer.

Contini M. (1992). Per una pedagogia delle emozioni. Firenze: La Nuova Italia.

Contini M. (2002). La comunicazione intersoggettiva fra solitudini e globalizzazioni. Milano: La Nuova Italia.

Contini M. \& Demozzi S. (2016). Corpi bambini. Sprechi d'infanzie. Milano: FrancoAngeli.

Corsi M., Fabbri M. \& Riva M. G. (a cura di) (2016). Educare le emozioni. Contro la violenza. Pedagogia Oggi, 1, 1-334.

Dambach K. E. (2009). Mobbing in der Schulklasse. München: Reinhardt Verlag.

Dato D. (2004). La scuola delle emozioni. Bari: Progedit.

Dato D. (2016). Emozioni e preoccupazione empatica. Una via pedagogica per imparare a prestare attenzione al mondo. In M. Corsi, M. Fabbri \& M. G. Riva (a cura di). Educare le emozioni. Contro la violenza (pp. 239-251). Pedagogia Oggi, 1, 1-334.

Dato D. (2019). Linsegnante emotivo. Formare tra mente e affetti. Bari: Progedit.

Dentith A. M., Redmon Wright R. \& Coryell J. (2015). Those mean girls and their friends: Bullying and mob rule in the academy. Adult Learning, 26(1), 28-34.

De Serio B. (2015). Il silenzio delle bambine e la lunga storia di un'invisibilità sociale. In D. Dato, B. De Serio \& A.G. Lopez (a cura di). La formazione al femminile. Itinerari storico-pedagogici. Bari: Progedit.

Dozza L. (a cura di) (2018). Io corpo Io racconto Io emozione. Bergamo: Zeroseiup.

Esch G. (2008). Children's Literature. Perceptions of Bullying. Childhood Education, 84(6), 379-382.

Fabbri M. (2008). Problemi d'empatia. La Pedagogia delle emozioni di fronte al mutamento degli stili educativi. Pisa: ETS. 
Fabbri M. (2014). Controtempo. Una duplice narrazione fra crisi ed empatia. Parma: Spaggiari.

Faeti A. (2001a). I diamanti in cantina. Come leggere la letteratura per ragazzi. Cesena: Il Ponte Vecchio.

Faeti A. (2001b). Le ortensie e le macerie. Infanzia in guerra. In E. Varrà (a cura di). L'età d'oro. Storie di bambini e metafore d'infanżia (pp. 29-46). Bologna: Pendragon.

Field J. E., Kobler J. B., Crothers L. M. \& Hughes T. L. (2009). Understanding Girl Bullying and what to Do About it. London: Sage.

Filograsso I. (2012). Bambini in trappola. Pedagogia nera e letteratura per l'infanzia. Milano: FrancoAngeli.

Fox C.L., Jones S.E., Stiff C.E. \& Sayers J. (2014). Does the Gender of the Bully/Victim Dyad and the Type of Bullying Influence Children's Responses to a Bullying Incident? Aggressive Behavior, 40, 359-368.

Frabboni F. (2012). Il problematicismo in pedagogia e in didattica. Trento: Erickson. Fratini T. (2015). Il bullismo in epoca di crisi. Una lettura pedagogica. Roma: Anicia. Genta M. S. (2002). Il bullismo. Bambini aggressivi a scuola. Roma: Carocci. Goleman D. (1996). Intelligenza emotive. Milano: Rizzoli.

Hinduja S. \& Patchin J. W. (2010). Bullying, cyberbullying, and suicide. Archives of Suicide Research, 14(3), 206-221.

Ladame F. (1987). I tentativi di suicidio degli adolescenti. Roma: Borla.

Loiodice I. (2004) (a cura di). Una scuola per tutti i bambini. I laboratori delle intelligenze e degli affetti nella scuola dell infanzia. Bari: Adda.

Loiodice I. \& Pinto Minerva F. (2015). Introduzione. In D. Dato, B. De Serio \& A.G. Lopez (a cura di). La formazione al femminile. Itinerari storico-pedagogici (pp. IX-XVIII). Bari: Progedit.

Mazzucco M.G. (2012). Prefazione. In B. Pitzorno. Ascolta il mio cuore. Milano: Mondadori.

McClung S.R. (2006). Cyberbullying: Mean girls or authentic relationships?, ProQuest Dissertations Publishing. St. Mary's University (Texas).

Morin E. (2000). La testa ben fatta. Riforma dell'insegnamento e riforma del pensiero. Milano: Raffaello Cortina.

Moulton E., Heath M.A., Prater M.A. \& Dyches T.T. (2011). Potrayals of Bullying in Children's Picture Books and Implications for Bibliotherapy. Reading Horizons, 51(2), 119-148.

Pietropolli Charmet G. (2004). Crisis Center. Il tentato suicidio in adolescenza. Milano: FrancoAngeli.

Rossi B. (2002). Pedagogia degli affetti. Roma-Bari: Laterza.

Scabello Garbin K. (2014). Oltre il confine invalicabile: il tema della finitudine nella letteratura per l'infanzia. In M. Campagnaro (a cura di). Le terre della fantasia. Leggere la letteratura per l'infanzia e l'adolescenza (pp. 147-201). Roma: Donzelli. 
Trisciuzzi M.T. (2016). Il futuro è per tutti. Il romanzo di formazione nelle opere di Marie-Aude Murail. Rivista di storia dell'educazione, 2, 313-322.

Trisciuzzi M.T. (2018a). Ritratti di famiglia. Immagini e rappresentazioni nella storia della letteratura per l'infanzia. Pisa: ETS.

Trisciuzzi M.T. (2018b). Bulle e pupe. Il bullismo femminile nella letteratura per l'infanzia e per ragazzi. In G. Burgio (a cura di). Comprendere il bullismo femminile. Genere, dinamiche relazionali, rappresentazioni (pp. 107-123). Milano: FrancoAngeli.

Van Gennep A. (2002). I riti di passaggio. Torino: Bollati Boringhieri.

Vegetti Finzi S. \& Battistin A.M. (2000). L'età incerta. I nuovi adolescenti. Milano: Mondadori.

Viljoen J.L., O’Neill M.L. \& Sidhu A. (2005). Bullying behaviors in female and male adolescent offenders: Prevalence, types and association with psychosocial adjustment. Aggressive Behavior, 31, 521-536.

Opere di narrativa

Arsenault I., \& Britt F. (2014). Jane, la volpe \& io. Milano: Mondadori.

Burnett F.H. (2013). Il giardino segreto. Milano: BUR.

Dahl R. (2003). Matilde [1988]. Milano: Salani.

Kelly J. \& Niimura J. M. K. (2010). I kill Giants. Milano: Bao Publishing. Murail M.-A. (2011). Nodi al pettine. Milano: Giunti.

Ness P. (2018). Questo libro. In S. Dowd. Il pavee e la ragazza. Crema: Uovonero.

Ness P., \& Dowd S. (2012). Sette minuti dopo la mezzanotte. Milano: Mondadori.

Paterson K. (2007). Un ponte per Terabithia. Milano: Mondadori.

Pitzorno B. (2012). Ascolta il mio cuore. Milano: Mondadori.

Smy P. (2017). Thornhill. Cremona: Uovonero. 